\title{
Korrektes Sponsoring von Fortbildungsanlässen: Eine «Checkliste» schafft Klarheit
}

Die Richtlinien «Zusammenarbeit Ärzteschaft-Industrie», welche die Schweizerische Akademie der Medizinischen Wissenschaften (SAMW) Ende 2005 verabschiedet und die FMH 2006 in ihre Standesordnung aufgenommen hat, haben noch nicht jenen «Kulturwandel» bei der Ärzteschaft bewirkt, den sich die Akademie erhofft hat. Noch immer gelten zahlreiche Anlässe als «Fortbildungsveranstaltungen», obwohl sie den Richtlinien teilweise in mehreren Punkten widersprechen.

Zahlreiche Fachgesellschaften (und auch die Arbeitsgruppe «Unterstützung der Fachgesellschaften im Bereich der Fortbildung» der früheren KWFB) haben wiederholt signalisiert, dass sie froh wären um eine Art «Checkliste», mit der geprüft werden könnte, ob ein Fortbildungsanlass den Richtlinien entspricht.

Die «Beratende Kommission» der SAMW, welche

Korrespondenz: SAMW Petersplatz 13 CH-4051 Basel mail@samw.ch die Umsetzung der Richtlinien «Zusammenarbeit Ärzteschaft-Industrie» begleitet und untersützt, hat dieses Anliegen aufgegriffen und einer Subkommission den Auftrag erteilt, den Entwurf einer solchen Checkliste auszuarbeiten. Die Subkommission hat den Entwurf im Rahmen einer kleinen Vernehmlassung mit VertreterInnen von FMH, SGAM, SGIM und SGPP diskutiert; die nun vorliegende Fassung ist das Resultat dieser Diskussionen. In der Folge hat die Beratende Kommission die «Checkliste» an ihrer Sitzung vom 24. Juni 2009 definitiv verabschiedet.

Die «Checkliste» enthält ein Titelblatt mit allen notwendigen Angaben zum geplanten Anlass sowie eine zweite Seite mit den zu erfüllenden Kriterien (siehe Kasten). Diese Checkliste ist vom Antragsteller bzw. der Antragstellerin auszufüllen und zusammen mit dem Antrag auf Credits bei der Fachgesellschaft einzureichen. Falls alle Fragen dieser Checkliste mit JA beantwortet werden, bedeutet dies, dass der Anlass den Richtlinien entspricht.

Die «Checkliste» wird im Sinne einer Hilfestellung allen Fachgesellschaften zur Verfügung gestellt; sie existiert auch in elektronischer Form und kann im Internet (www.samw.ch) heruntergeladen werden.

\section{Checkliste betreffend Vergabe von Credits für Fortbildungs- Veranstaltungen durch medizinische Fachgesellschaften[1]}

Die Beratungskommission[2] für die Umsetzung der Richtlinien Ärzteschaft-Industrie [3] empfiehlt den medizinischen Fachgesellschaften, beim Entscheid über die Vergabe von Credits für Fortbildungs-Veranstaltungen nach folgender Checkliste vorzugehen. Eine Veranstaltung kann nur dann Credits erhalten, wenn sie:

- sich an den Zielen der Fortbildungsordnung (FBO) der FMH (und den Fortbildungsprogrammen der Fachgesellschaften orientiert sowie

- die folgenden, an den SAMW-Richtlinien Ärzteschaft-Industrie (Ziffer II) orientierten Anforderungen erfüllt.

Zur Vermeidung administrativer Umtriebe können die Fachgesellschaften regelmässig durchgeführte, eigene Fortbildungs-Veranstaltungen oder solche von Spitälern bzw. Spitalabteilungen en bloc bzw. im Voraus anerkennen. Voraussetzung dafür ist die schriftliche Zusicherung der betreffenden Fachgesellschaft bzw. der Spitäler und Spitalabteilungen, dass diese Fortbildungs-Veranstaltungen den nachfolgenden Kriterien entsprechen.

\section{Veranstaltung}

Bezeichnung:

Datum:

Ort:

Gemäss der Fortbildungswww.fmh.ch/bildungsiwf/fortbildung.html

2 www.samw.ch/dms/de/ Ethik/RL/AG/d_Umsetzung _RL_Ae_ind.pdf

3 www.samw.ch/dms/de/ Ethik/RL/AG/d_RL_ZAeI_ 06.pdf

41 Credit entspricht einer Stunde
Effektive Dauer der Fortbildung (ohne Pausen, ohne Rahmenprogr.): Beantragte Anzahl Credits [4]:

\section{Antragsteller}

Titel:

Vorname: $\quad$ Name:

Funktion: Institution:

vertreten durch (nichtärztliches Kongress-Organisationsunternehmen): allenfalls:

PLZ, Ort:

Tel.:

E-Mail:

$\rightarrow$ Erläuterungen zu allfälligen Nein-Antworten auf der Checkliste (bitte Checklisten-Nummer angeben): 
Kriterien für die Anerkennung als Fortbildungs-Veranstaltung

1. Die Veranstalter sind in freier Praxis oder in einem Spital tätige Ärzte oder ein ärztliches Fachgremium (d. h. nicht ein Industrieunternehmen).

Veranstalter sind grundsätzlich im jeweiligen Fachgebiet kompetente medizinische Fachorganisationen, Institutionen oder Fachpersonen und nicht Unternehmen der Industrie oder anderer kommerzieller Anbieter oder Dienstleister, die Veranstaltungen unterstützen (sponsern).

2. Die Veranstalter bestimmen den Inhalt (inkl. Berücksichtigung EBM, Wirtschaftlichkeit, Objektivität), die Referenten und den Ablauf der Veranstaltung selbständig, d.h. unabhängig von unterstützenden (sponsernden) Unternehmen.

Die Veranstalter bestimmen das Programm (Inhalt und Ablauf) und die Referenten, und nicht die Unternehmen, welche die Veranstaltung unterstützen (sponsern).

In den Referaten und Diskussionen sollen die Themen objektiv nach dem aktuellen Stand der wissenschaftlichen Erkenntnis und von verschiedenen Seiten her (interdisziplinär) behandelt werden. Die Diagnose- und Therapiemöglichkeiten sollen in der Regel vollständig und so weit wie möglich nach den Kriterien der EBM dargestellt werden.

In den Referaten sollen Arzneimittel grundsätzlich mit der international anerkannten Wirkstoffbezeichnung «generic name» erwähnt werden.

3. Das allenfalls an der Veranstaltung gebotene Rahmenprogramm ist gegenüber dem fachlichen Teil eindeutig untergeordnet.

Mindestens $80 \%$ der Zeit der Veranstaltung sind für den Fachteil bestimmt; der finanzielle Aufwand für die gesamte Veranstaltung spiegelt dieses Verhältnis wider. Rahmenprogramm und Fachteil müssen klar getrennt sein.

4. Falls die Veranstaltung von privaten Unternehmen finanziell unterstützt (gesponsert) wird, sind mehrere Unternehmen an diesem Sponsoring beteiligt (kein Monosponsoring).

Als Sponsoring fallen namentlich finanzielle Beiträge sowie organisatorische oder logistische Unterstützung in Betracht. Allfällige Nein-Antwort bitte auf der Vorderseite begründen.

5. Die Einzelheiten der Unterstützung der Veranstaltung sind in einer schriftlichen Vereinbarung zwischen den Veranstaltern und unterstützenden (sponsernden) Unternehmen festgehalten, und die finanziellen Mittel für die Veranstaltung aus dem Sponsoring werden auf ein dafür bestimmtes Konto des Veranstalters verbucht.

Die Veranstalter legen der Fachgesellschaft die Vereinbarungen auf Anfrage zur Einsicht vor. In Spitälern stattfindende ganz- oder mehrtägige Veranstaltungen, die von Unternehmen der Industrie unterstützt (gesponsert) werden, sind von der Abteilungs- resp. Spitalleitung oder von der sonst dafür zuständigen Stelle zu genehmigen. Die Kontrolle der Finanzen ist Sache der Veranstalter. Die Veranstalter legen den Sponsoren und den Fachgesellschaften Budget und Rechnung auf Anfrage vor.

6. Die an der Veranstaltung als Zuhörer (d.h. ohne Beteiligung mit Referat oder Poster) teilnehmenden Ärzte leisten eine angemessene Kostenbeteiligung.

Im Interesse ihrer Unabhängigkeit bezahlen die Teilnehmer einer Veranstaltung eine Teilnahmegebühr sowie einen angemessenen Beitrag an die Reise- und Unterkunftskosten.

Die Bemessung der Kostenbeteiligung richtet sich vor allem nach der Dauer der Veranstaltung und berücksichtigt die berufliche Stellung des Arztes (d.h. geringere Kostenbeteiligung für ÄrztInnen in Weiterbildung möglich).

Die Kosten für zusätzliche Hotelaufenthalte, Reisen oder andere Aktivitäten, die mit der Veranstaltung keinen inhaltlichen Zusammenhang haben, gehen vollumfänglich zulasten der Teilnehmer bzw. allfälliger Begleitpersonen.

7. Es ist sichergestellt, dass die Referenten und Veranstalter allfällige persönliche oder institutionelle, kommerzielle Interessen, finanzielle Verbindungen zum Sponsor, Beratertätigkeit im Auftrag des Sponsors oder Forschungsunterstützung durch den Sponsor offenlegen. Die Einladung an die ÄrztInnen erfolgt frei von Werbung für den oder die Sponsor(en) oder ihre Produkte.

Im Programm und in den Unterlagen einer Veranstaltung werden alle Sponsoren aufgeführt. Referenten legen ihre Interessensbindungen dem Veranstalter, der Fachgesellschaft sowie vor Beginn ihrer Präsentation den Teilnehmern auf geeignete Weise offen.

Datum:

Unterschrift des Antragstellers: 